\title{
Correctors of the Major Cystic Fibrosis Mutant Interact through Membrane-Spanning Domains ${ }^{\text {[ }}$
}

\author{
Onofrio Laselva, Steven Molinski, Valeria Casavola, and Christine E. Bear
}

Programme in Molecular Medicine, Hospital for Sick Children, Toronto, Ontario, Canada (O.L., S.M., C.E.B.); Department of Biosciences, Biotechnologies and Biopharmaceutics, University of Bari, Bari, Italy (V.C.); and Departments of Biochemistry and Physiology, University of Toronto, Toronto, Ontario, Canada (C.E.B.)

Received January 22, 2018; accepted March 22, 2018

\begin{abstract}
The most common cystic fibrosis causing mutation is deletion of phenylalanine at position 508 (F508del), a mutation that leads to protein misassembly with defective processing. Small molecule corrector compounds: VX-809 or Corr-4a (C4) partially restores processing of the major mutant. These two prototypical corrector compounds cause an additive effect on F508del/cystic fibrosis transmembrane conductance regulator (CFTR) processing, and hence were proposed to act through distinct mechanisms: VX-809 stabilizing the first membrane-spanning domain (MSD) 1, and C4 acting on the second half of the molecule [consisting of MSD2 and/or nucleotide binding domain (NBD) 2]. We confirmed the effect of VX-809 in enhancing the stability of MSD1 and showed that it also allosterically modulates MSD2 when coexpressed with MSD1. We showed for the first time that C4 stabilizes the second half of the CFTR protein through its
\end{abstract}

action on MSD2. Given the allosteric effect of VX-809 on MSD2, we were prompted to test the hypothesis that the two correctors interact in the full-length mutant protein. We did see evidence supporting their interaction in the full-length F508del-CFTR protein bearing secondary mutations targeting domain:domain interfaces. Disruption of the MSD1:F508delNBD1 interaction (R170G) prevented correction by both compounds, pointing to the importance of this interface in processing. On the other hand, stabilization of the MSD2: F508del-NBD1 interface (by introducing R1070W) led to a synergistic effect of the compound combination on the total abundance of both the immature and mature forms of the protein. Together, these findings suggest that the two correctors interact in stabilizing the complex of MSDs in F508del-CFTR.

\section{Introduction}

Cystic fibrosis (CF) is caused by mutations in the $\mathrm{CF}$ transmembrane conductance regulator (CFTR) CFTR / ABCC7 gene (Riordan et al., 1989; Gadsby et al., 2006; Molinski et al., 2012). The CFTR protein functions as an ATP- and phosphorylation-regulated chloride channel comprised of two membrane-spanning domains (MSDs), MSD1 and MSD2 [with a total of four intracellular loops (ICLs): ICL1-4]; two nucleotide binding domains (NBDs), NBD1 and NBD2; and a regulatory domain (Riordan, 2008; Molinski et al., 2012). The physiologic role of the CFTR channel is for maintenance of fluid transport across epithelial cells of the body's tubular organs, including the airways, intestines, and pancreatic as well as bile ducts, and loss of this function causes $\mathrm{CF}$ with associated multisystem pathologies (Huang et al., 2004; Li and Naren, 2005).

Although over 2000 CFTR mutations have been described in the literature and mutation databases, most research efforts

This work was supported by Cystic Fibrosis Canada and the Canadian Institute of Health Research [Grants CIHR MOP-97954 and CIHR GPG102171] (to C.E.B.).

https://doi.org/10.1124/mol.118.111799.

S This article has supplemental material available at molpharm. aspetjournals.org. have been focused on $\mathrm{CF}$ patients bearing the most common disease-causing mutations: deletion of phenylalanine at position 508 (F508del) and the glycine to aspartate missense mutation at position 551 (G551D) (Riordan et al., 1989; Gadsby et al., 2006; Cutting, 2015). For more information, see the CFTR1 (http://www.genet.sickkids.on.ca) and CFTR2 (http://cttr2.org/) databases. F508del causes aberrant assembly of the full-length protein, which is mediated through the NBD1:ICL4 interface. Misassembly caused by F508del impairs processing, trafficking, and stability of the full-length protein at the cell surface, while G551D causes a channel gating defect due to aberrant NBD1:NBD2 interdomain interaction (Mornon et al., 2008; Mendoza et al., 2012; Rabeh et al., 2012). However, two small molecule-based therapies, ORKAMBI and KALYDECO, have recently been developed for patients bearing F508del and G551D, respectively. KALYDECO, also known as ivacaftor (VX-770), is a small molecule potentiator that promotes the open state of G551D-CFTR channels, while ORKAMBI is comprised of both VX-770 and lumacaftor (VX-809), a small molecule corrector that promotes protein stability and forward trafficking of F508del-CFTR (Van Goor et al., 2009, 2011).

The mechanism of action of VX-809 (recently categorized as a class I corrector) has been interrogated by several groups, yet

ABBREVIATIONS: CF, cystic fibrosis; CFTR, cystic fibrosis transmembrane conductance regulator; $\mathrm{CH}$, coupling helix; DMSO, dimethylsulfoxide; F508del, deletion of phenylalanine at position 508; G551D, glycine to aspartate missense mutation at position 551; HEK, human embryonic kidney; ICL, intracellular loop; MSD, membrane-spanning domain; NBD, nucleotide binding domain; TMA, 4,6,4'-trimethylangelicin; WT, wild type. 
uncertainties remain (Loo et al., 2013; Ren et al., 2013; Laselva et al., 2016; Hudson et al., 2017; Loo and Clarke, 2017). Previous studies using isolated domains of CFTR showed that the N-terminal domain of CFTR, MSD1, was important for the stabilizing effect of VX-809 (Loo et al., 2013; Ren et al., 2013). In the context of the full-length mutant protein: the F508del-CFTR bearing strategically substituted cysteines at the interface between NBD1 and the coupling helix of MSD2, and VX-809 promoted chemical crosslinking, supporting the hypothesis that modification of this interdomain interface constituted its major mechanism of action (Loo and Clarke, 2017). However, it is not clear how the interaction of VX-809 with MSD1 mediates such long-range interdomain conformational changes involving MSD2.

Another pharmacological corrector (class II), Corr-4a (C4), also partially ameliorate the processing defect of F508del-CFTR, and since its rescue effects are additive with VX-809, C4 is thought to act via a distinct mechanism (Okiyoneda et al., 2013). Okiyoneda et al. (2013) suggested that the $\mathrm{C} 4$ binding site may be located in NBD2; however, the binding site, as well as the intramolecular consequences on the CFTR structure/function of this small molecule has not yet been defined (Okiyoneda et al., 2013).

The goal of the current study was to provide insight into the mechanism of action of VX-809 and C4. We used several experimental approaches toward this goal, including the use of CFTR fragments to define the domains modified by these small molecules and studies of the full-length F508del-CFTR bearing interface-modifying amino acid substitutions.

\section{Materials and Methods}

Cell Culture and Transfection. All CFTR variants and fragments used in this study were transiently expressed in human embryonic kidney (HEK) GripTite cells, HEK293 (a gift from Dr. Daniela Rotin, Hospital for Sick Children). HEK293 cells were maintained in Dulbecco's modified Eagle's medium (Wisent, St-Bruno, QC, Canada) supplemented with nonessential amino acids (Life Technologies, Waltham, MA) and 10\% fetal bovine serum (Wisent) at $37^{\circ} \mathrm{C}$ and processed with $5 \% \mathrm{CO}_{2}$ as previously described (D'Antonio et al., 2013; Molinski et al., 2015). Transient transfections were performed using PolyFect Transfection Reagent (Qiagen, Hilden, Germany) according to the manufacturer's protocol, as previously described (Molinski et al., 2015).

Plasmids, Antibodies, and Reagents. Briefly, mutant CFTR constructs were generated using the KAPA HiFi HotStart PCR Kit (KAPA BIOSYSTEMS, Woburn, MA) according to the manufacturer's standard polymerase chain reaction protocol with high-quality ( $>300 \mathrm{ng} / \mu \mathrm{l}, 260 / 280 \mathrm{~nm}$ ratio of 1.8 ) plasmid DNA containing wildtype (WT)-CFTR of F508del-CFTR cDNA (in pcDNA3.1) as the template, as previously described (Molinski et al., 2015). The primary antibodies used in this study were mAb MM13-4 (EMD Millipore, Billerica, MA) for MSD1-containing constructs, mAb A52 (a kind gift from Dr. David Clarke, University of Toronto) (Loo et al., 2013) for MSD2-containing constructs, and mAb 596 (University of North Carolina at Chapel Hill, Chapel Hill, NC) for NBD2-containing constructs (Cui et al., 2007). Calnexin was used as a protein loading control and detected with a calnexin-specific rabbit pAb (SigmaAldrich, St. Louis, MO). The small molecule modulators of CFTR used in this study were VX-770 and VX-809 (Selleck Chemicals, Houston, TX), 4,6,4'-trimethylangelicin (TMA) (Dr. Roberto Gambari, University of Ferrara, Italy) (Favia et al., 2014; Abbattiscianni et al., 2016), C4 and CFTR-specific inhibitor 172 (CFTRinh-172; Cystic Fibrosis Foundation Therapeutics, Bethesda, MD), and glycerol (SigmaAldrich).

Immunoblotting. For the purpose of CFTR immunoblotting, transfected cells were lysed in modified radioimmunoprecipitation assay buffer containing a protease inhibitor cocktail (Roche, Mannheim, Germany) for 10 minutes. Soluble fractions were analyzed by SDS-PAGE on 6\% Tris-Glycine Gels (Life Technologies). After electrophoresis, proteins were transferred to nitrocellulose membranes (Bio-Rad, Hercules, CA), incubated in 5\% milk, and CFTR bands were detected with human CFTR-specific murine mAb 596 (1:5000). The blots were developed with either enhanced chemiluminescence (Amersham, Buckinghamshire, UK) and exposure to film for 0.5-5 minutes as required, or with enhanced chemiluminescence (Amersham) on the Li-Cor Odyssey Fc (LI-COR Biosciences, Lincoln, NE) in a linear range of exposure (Laselva et al., 2016; Molinski et al., 2017).

Measurement of CFTR Fragment Stability and Glycosylation Status. Steady-state levels of CFTR fragments, including MSD1 (containing residues 1-380, or K381X), A52-tagged MSD2 (containing residues 837-1196), and MSD2-NBD2 (containing residues 8501480), were determined by western blot analysis. HEK293 cells were transiently transfected with the plasmids as described previously. After 18 hours of transfection, the cells were treated with $3 \mu \mathrm{M}$ VX-809, 500 nM TMA, $10 \mu \mathrm{M}$ C4, or vehicle control [dimethylsulfoxide (DMSO)], and 24 hours after correction the cells were lysed in modified radioimmunoprecipitation assay buffer $(50 \mathrm{mM}$ Tris- $\mathrm{HCl}, 150 \mathrm{mM}$ $\mathrm{NaCl}, 1 \mathrm{mM}$ EDTA, pH 7.4, 0.2\% SDS, and 0.1\% Triton X-100) containing a protease inhibitor cocktail (Roche) for 10 minutes. Then, the soluble fractions were analyzed by SDS-PAGE on 4\%-12\% TrisGlycine Gels (Life Technologies). After electrophoresis, proteins were transferred to nitrocellulose membranes (Bio-Rad), incubated in 5\% milk, and CFTR bands were detected with human CFTR-NBD2specific murine mAb 596 (1:10,000), human CFTR-MSD1-specific murine mAb MM13-4 (1:10,000), or monoclonal antibody A52 for MSD2 (1:1000) (Dr. David Clarke, University of Toronto) (Loo et al., 2013; Molinski et al., 2015; Laselva et al., 2016).

To evaluate protein glycosylation status of MSD2 we first transfected HEK293 cells, and then after 48 hours the cells were lysed with modified radioimmunoprecipitation assay buffer as described previously. Lysates were treated with either endoglycosidase $\mathrm{H}$ or peptide-N-glycosidase F (both from New England Biolabs, Ipswich, MA) according to the manufacturer's protocol. Western blots were obtained using the murine monoclonal antibody A52 as described by Loo et al. (2013). The blots were developed with enhanced chemiluminescence (Amersham) and exposure to film for 0.5-5 minutes as required. Relative expression levels of CFTR proteins were quantitated by densitometry of western blots using ImageJ software version 1.46 (National Institutes of Health, Bethesda, MD).

Cycloheximide Chase Assay. To test the effect of C4 on the stability of MSD1, MSD2, or MSD2-NBD2 fragments expressed as separate polypeptides, HEK293 cells were transfected as described previously, and after 18 hours medium was replaced with fresh medium containing either vehicle (DMSO) or $\mathrm{C} 4(10 \mu \mathrm{M})$. After 24 hours at $27^{\circ} \mathrm{C}$, protein synthesis was stopped by addition of medium containing $0.5 \mathrm{mg} / \mathrm{ml}$ cycloheximide and either vehicle (DMSO) or C4 $(10 \mu \mathrm{M})$. The cells were then incubated at $37^{\circ} \mathrm{C}$, lysed at various time periods $(0-8$ hours), and whole cell extracts were subjected to western blot analysis as described previously (Cui et al., 2007; Loo et al., 2013).

Statistical Analysis. Data are represented as mean \pm S.D. unless otherwise noted. GraphPad Prism 7.0 software (GraphPad, San Diego, CA) was used for all statistical tests. One-way/two-way analyses of variance were conducted as appropriate, and $P<0.05$ was considered significant. Data with multiple comparisons were assessed with Tukey's multiple-comparisons test with $\alpha=0.05$. Each experiment is defined as a separate biologic study on independently plated cells.

\section{Results}

VX-809 and C4 Differentially Modify the Stability of MSD1 and MSD2. It has previously been reported that the class I corrector VX-809 stabilizes a protein fragment comprising MSD1 (residues 1-380) of CFTR but not domains 
comprising the second half of the CFTR protein (i.e., MSD2NBD2, residues 850-1480) (Ren et al., 2013; Laselva et al., 2016). These results were confirmed in the present study (Fig. 1A, panels i and ii). However, since certain reports showed that VX-809 enhanced the interaction between MSD2 and F508del-NBD1 in the context of the full-length mutant protein, we hypothesized that VX-809 may also modulate the second half of the protein if coexpressed with MSD1. Interestingly, when coexpressed with MSD1, the steady-state abundance of MSD2-NBD2 was significantly increased by $3 \mu \mathrm{M}$ VX-809 (Fig. 1A, panels i and iii). Similarly, when coexpressed with MSD1, the steady-state abundance of MSD2 was significantly increased by VX-809 (Fig. 1B, panels i and iii). These data suggest that VX-809 allosterically modifies the stability of MSD2 through its interaction with MSD1.

We then assessed the effect of $10 \mu$ Corr4a (C4) on fragment stability in a similar set of studies. In contrast to VX-809, C4 treatment stabilized the MSD2-NBD2 fragment rather than MSD1 (Fig. 2A, panels i and ii). Interestingly, there was no positive (i.e., allosteric) effect of C4 on MSD1 when coexpressed with MSD2-NBD2. We interpret these findings to suggest that C4 interacts with the second half of CFTR and this interaction does not indirectly modify the abundance of MSD1.

To test the hypothesis that C4 binding to MSD2-NBD2 enhances its stability, we conducted cycloheximide-chase experiments, wherein protein synthesis was inhibited after production of this fragment, and then the abundance monitored over time in the presence or absence of $\mathrm{C} 4$. We observed a modest increase in the stability of this fragment in the presence of $\mathrm{C} 4$ at 1 hour after initiation of the chase (Supplemental Fig. 1).

To determine if $\mathrm{C} 4$ binds to the MSD2 component of this two-domain fragment, we studied the effect of C4 on MSD2 alone. We transfected HEK cells with MSD2 bearing an A52 tag, and after treatment with $\mathrm{C} 4$ for 24 hours (see Materials and Methods) this CFTR fragment was expressed as three distinct bands (Fig. 3A). As indicated by the sensitivity to both endoglycosidase $\mathrm{H}$ and peptide- $\mathrm{N}$-glycosidase $\mathrm{F}$, the higher migrating species of MSD2 represent differentially glycosylated, high mannose forms of this domain (Du and Lukacs, 2009). Next, to determine if C4 interacts with MSD2, we preincubated HEK cells expressing MSD2 with $3 \mu \mathrm{M}$ VX-809, $500 \mathrm{nM}$ TMA (a compound thought to act similarly to VX-809), $10 \mu \mathrm{M} \mathrm{C} 4$, or vehicle control (DMSO), and found that only C4 significantly increased its abundance (Fig. 3B).

In Fig. 3, $\mathrm{C}$ and $\mathrm{D}$, we show that in the absence of $\mathrm{C} 4$ the half-life of MSD1 and MSD2 is short after cyclohexamide addition. Interestingly, however, treatment with $\mathrm{C} 4$ significantly increased the abundance of the MSD2 fragment but not the MSD1 fragment 2 hours after initiation of the cycloheximide-chase, supporting the hypothesis that C4dependent stabilization of CFTR is mediated via its interaction with MSD2.

Interaction between VX-809 and C4 Revealed in FullLength F508del-CFTR Bearing MSD:NBD1 Interface Mutants. Our findings that VX-809 stabilizes MSD2 allosterically via MSD1 and C4 also stabilizes MSD2 led us to hypothesize that the two correctors may interact to modulate the full-length F508del-CFTR protein. However, previous (and the current studies) (Fig. 4A), suggest that VX-809 $(3 \mu \mathrm{M})$ and $\mathrm{C} 4(10 \mu \mathrm{M})$ may not interact since their combined effect on F508del-CFTR abundance and processing

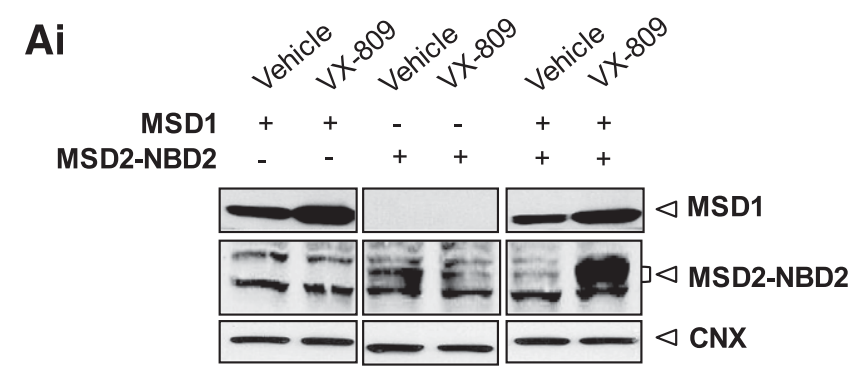

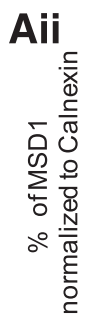
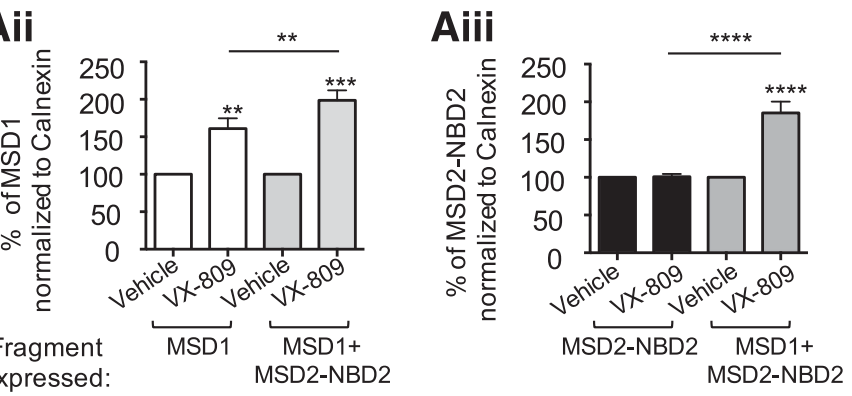

$\mathrm{Bi}$
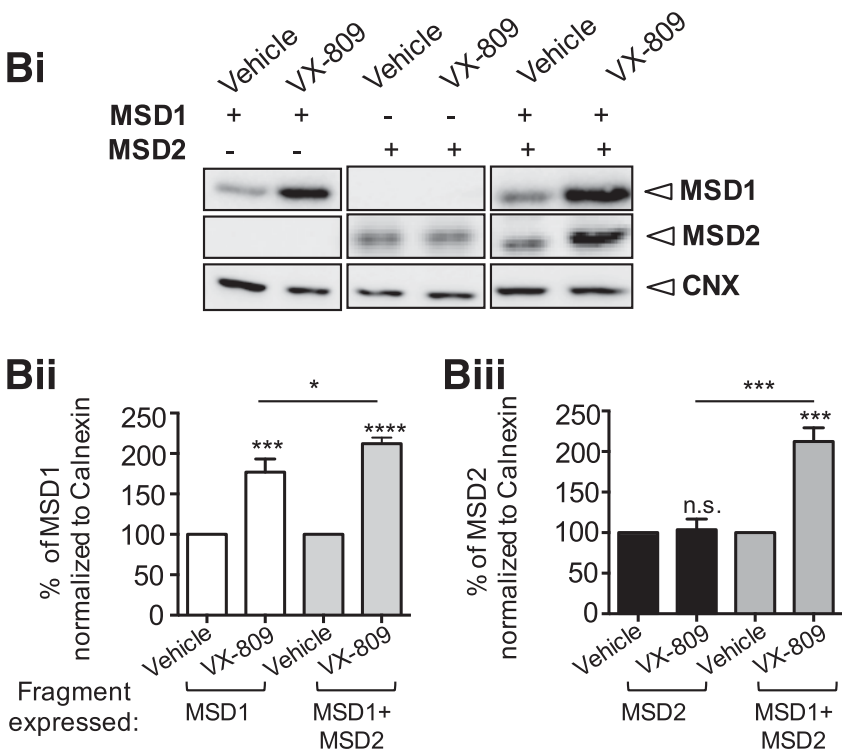

Fig. 1. Class I corrector VX-809 stabilizes the interaction between MSD1 and MSD2. (A, panel i) HEK293 cells were transiently transfected with MSD1 or MSD2-NBD2, or cotransfected with MSD1 and MSD2-NBD2 domains. (B, panel i) HEK293 cells were transiently transfected with MSD1 or MSD2, or cotransfected with MSD1 and MSD2 domains. Cells were treated for 24 hours at $37^{\circ} \mathrm{C}$ with DMSO, VX-809 $(3 \mu \mathrm{M})$. The MSD1 fragment was detected with an antibody against the N-terminus of CFTR (i.e., mAb MM13-4), and the MSD2-NBD2 fragment was detected with an antibody against the C-terminus of CFTR (mAb 596) and MSD2 fragment with antibody A52. (A, panel ii) Bar graphs show the mean ( \pm S.E.M.) of the percentage of abundance of MSD1 normalized to calnexin loading. Data are representative of four biologic studies $(* * P<0.01$; $* * * P<0.001)$. (A, panel iii) Bar graphs show the mean ( \pm S.E.M.) of the percentage of abundance of MSD2-NBD2 normalized to calnexin loading. Data are representative of four biologic studies (****P<0.0001). (B, panel ii) Bar graphs show the mean ( \pm S.E.M.) of the percentage of abundance of MSD1 normalized to calnexin loading. Data are representative of four experiments $(* P<0.05$; *** $P<0.001$; **** $P<0.0001)$. (B, panel iii) Bar graphs show the mean ( \pm S.E.M.) of the percentage of abundance of MSD2 normalized to calnexin loading. Data are representative of four biologic studies $(* * * P<0.001)$. n.s., not significant.

was additive, i.e., not supporting cooperativity. We were prompted to determine if such interaction would be revealed in F508del-CFTR proteins bearing secondary mutations in 
Ai

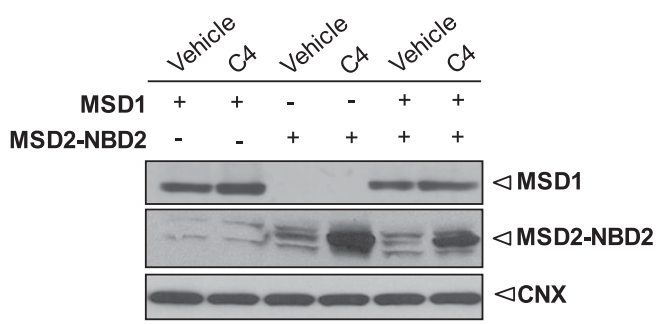

Aii

MSD1 Abundance

Aiii

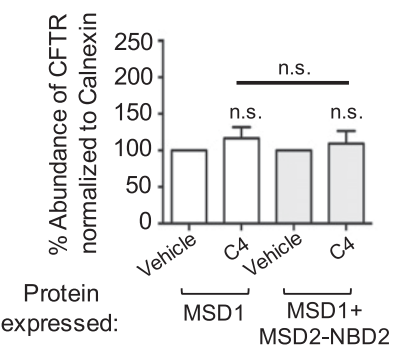

mature (band C) CFTR protein product (Okiyoneda et al., 2013). Both VX-809 and C4 improved processing of R170GCFTR, and together exerted an additive effect on processing to band C (Supplemental Fig. 2). On the other hand, R170G/F508del-CFTR failed to show correction of processing in response to either corrector alone or in combination, emphasizing the important function of $\mathrm{CH} 1$ in the F508del assembly. Interestingly, the abundance of the core glycosylated form (i.e., the band B form) of the CFTR protein was increased synergistically by the corrector combination, suggesting that these compounds interact with the full-length protein to improve stability (as in the case of MSD fragment studies) yet fail to induce improved assembly of F508delCFTR when the CH of ICL1 is disrupted (Fig. 4B).

We were then prompted to study the consequences of corrector compounds alone and in combination in F508delCFTR bearing a rescue mutation: $\mathrm{R} 1070 \mathrm{~W}$, located in the $\mathrm{CH}$ of ICL4. As previously shown (He et al., 2013; Okiyoneda et al., 2013), we confirmed that VX-809 and C4 rescued the processing of F508del/R1070W-CFTR individually and their effect on processing was additive (Fig. 4C; Supplemental Fig. 3). Interestingly, and consistent with the concept that both correctors mediate interacting effects on protein stability, we observed a synergistic effect of VX-809 plus C4 on the steadystate abundance of both the immature and mature forms of the F508del-CFTR-R1070W protein (Fig. 4C). These findings are consistent with the concept that the two compounds interact to promote protein stability.

\section{Discussion}

regions previously shown to modulate the assembly and correction of the major mutant.

As previously reported, the disease-causing R170G mutation in coupling helix $(\mathrm{CH}) 1$ (CH1-extended from MSD1 to interact with NBD1) impairs processing of WT-CFTR to a

In this study, we showed that two corrector compounds, prototypical of classes I and II, modify different regions of CFTR protein as previously suggested by Grove et al. (2009) and Okiyoneda et al. (2013). When individual domains were studied independently, VX-809 treatment stabilized MSD1,
A

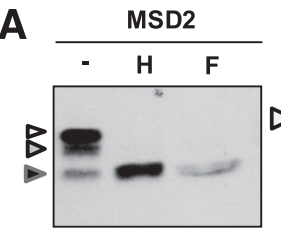

B
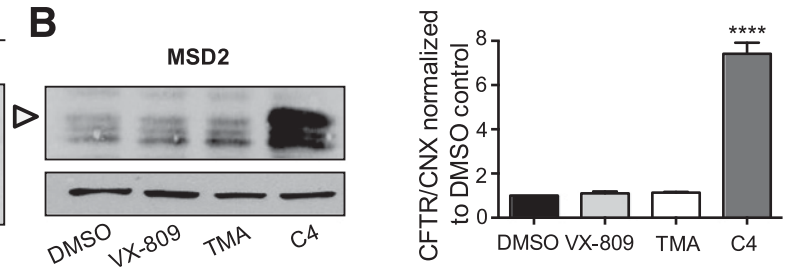

C

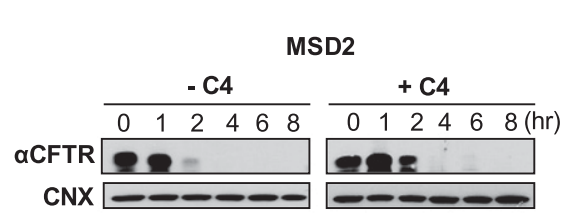

D

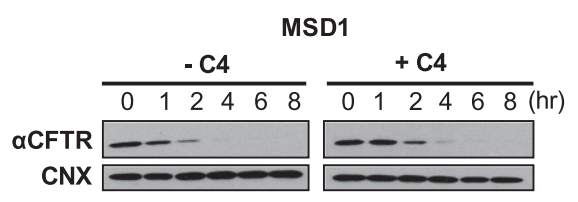

Ci

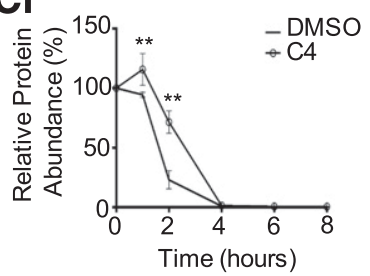

$\mathrm{Di}$

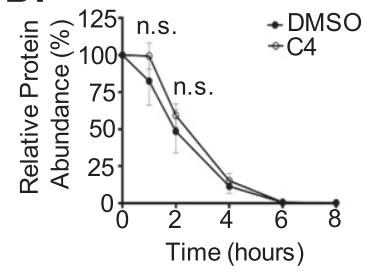

Fig. 3. C4 directly stabilizes MSD2 of CFTR. (A) Endoglycosidase sensitivity of the MSD2-CFTR fragment. Whole cell extracts of HEK293 cells transfected with A52-tagged MSD2-CFTR cDNA were treated with endoglycosidase $\mathrm{H}$ $(\mathrm{H})$, peptide-N-glycosidase $\mathrm{F}(\mathrm{F})$, or untreated (-). (B) Effect of C4 $(10 \mu \mathrm{M})$, TMA (500 nM), or VX-809 $(3 \mu \mathrm{M})$ on the abundance of A52-tagged MSD2-CFTR in HEK293 cells. (C and D) MSD1 or MSD2 domains of CFTR were expressed in HEK293 cells in the presence or absence of C4 $(10 \mu \mathrm{M})$ at $27^{\circ} \mathrm{C}$. After 24 hours, protein synthesis was inhibited by addition of cycloheximide $(0.5 \mathrm{mg} / \mathrm{ml})$ with or without $\mathrm{C} 4$ $(10 \mu \mathrm{M})$, and cells were collected after the indicated times for western blot analysis of whole cell extracts. (C, panel i; and D, panel i) The amount of CFTR protein at each time point was quantitated and expressed relative to that at time 0 and calnexin loading. Data are representative of three biologic studies $(* * P<0.01)$. 


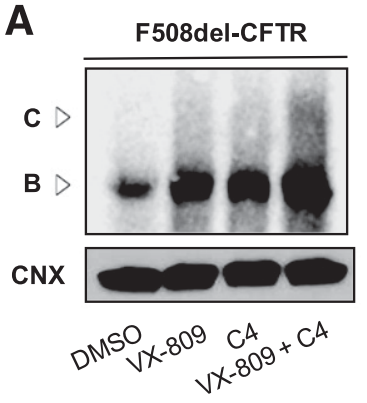

B

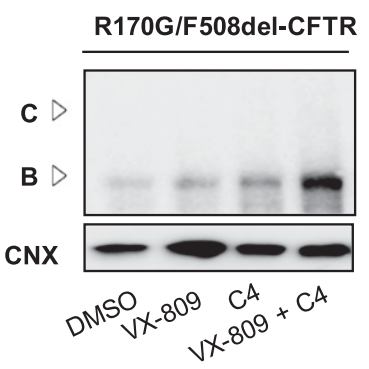

C

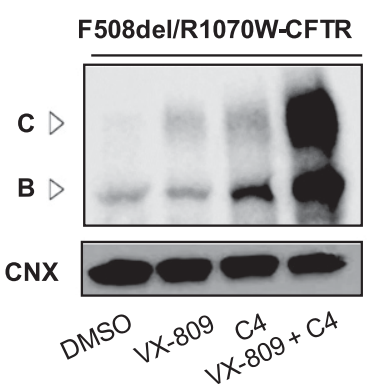

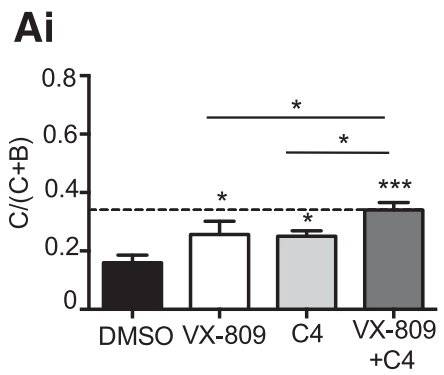

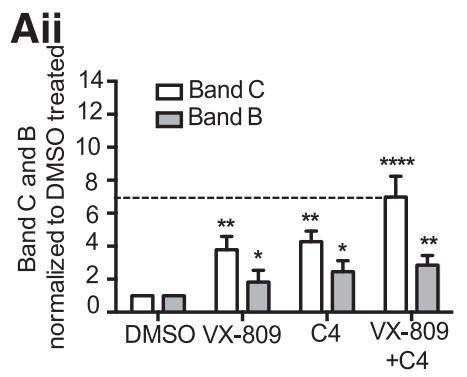

Bi

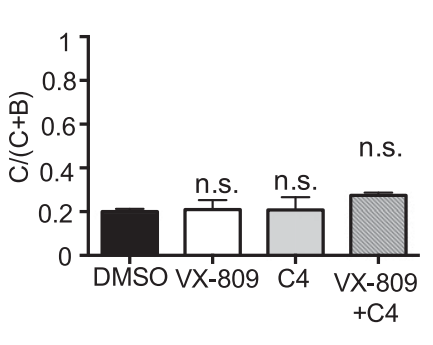

Bii

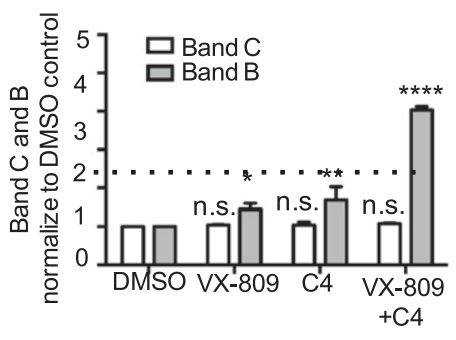

$\mathbf{C i}$

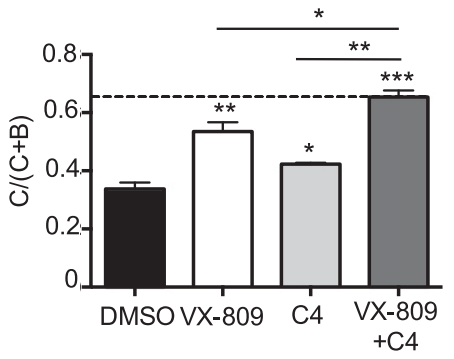

\section{Cii}

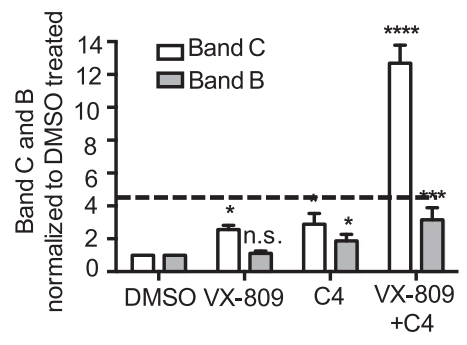

Fig. 4. Additive effect of VX-809 and C4 on correction of F508del-CFTR. (A-C) HEK293 cells were transiently transfected with F508del-CFTR, R170G/F508del, or F508del/R1070WCFTR, and treated for 24 hours at $37^{\circ} \mathrm{C}$ with DMSO, VX-809 $(3 \mu \mathrm{M})$ and/or C4 $(10 \mu \mathrm{M})$. F508del-CFTR, R170G/F508del-CFTR, and F508del/ R1070W-CFTR were detected with an antibody against the $\mathrm{N}$-terminus of CFTR (MM13-4). (C) Mature, complex-glycosylated CFTR; (B) immature, core-glycosylated CFTR. (A, panel i; B, panel i; and C, panel i) Bars represent the mean ( \pm S.E.M.) of the ratio $\mathrm{C} /(\mathrm{C}+\mathrm{B})$ and are representative of three biologic studies $(* P<0.05$; $* * P<0.01$; $* * * P<0.001$ ). (A, panel ii; $\mathrm{B}$, panel ii; and $\mathrm{C}$, panel ii) Bars represent the $\mathrm{B}+\mathrm{C}$ and band $\mathrm{B}$ forms, normalized to DMSO and are representative of three biologic studies $(* P<0.05 ; * * P<0.01 ; * * * P<0.001$; $* * * * P<0.0001)$. Stippled horizontal lines in (A and $\mathrm{C})$ show prediction for additive effect of VX-809 plus $\mathrm{C} 4$ on $\mathrm{C} / \mathrm{C}+\mathrm{B}$ (left bar graph) or band $\mathrm{C}$ (right bar graph). Dotted horizontal line in (B) shows prediction for additive effect on band B (right bar graph). n.s., not significant. whereas C4 treatment stabilized MSD2. Interestingly, we also report the first evidence that there is an overlapping effect of the two compounds on the stability of the MSDs when they are coexpressed. VX-809 exerted an allosteric effect on MSD2, supporting a new hypothesis wherein the two compounds exert an interacting mechanism of action (Fig. 5). The interacting effect of the two compounds was revealed in studies of full-length F508del-CFTR bearing MSD:NBD1 interface mutants.

The findings in the current work support several previous publications regarding the mechanism of action of class I and II corrector compounds. Grove et al. (2009) were the first to show that the class I compound VX-809 enhanced the stability of a fragment of CFTR corresponding to MSD1. Recent publications by Loo et al. (2013) and Loo and Clark (2017) similarly showed that VX-809 corrector activity is dependent on MSD1. We confirmed these findings, and furthermore showed that VX-809 treatment also stabilized the MSD2 domain fragment but only when coexpressed with MSD1. Our results provide greater understanding of the mechanism underlying the well-described modulation by VX-809 on the interaction between ICL4 (MSD2) and NBD1 in the full-length mutant protein, namely, by promoting a stable MSD1:MSD2 complex.

Previous studies of the mechanism of action of $\mathrm{C} 4$ (the prototypical class II corrector) supported a role for direct interaction with F508del-CFTR (Grove et al., 2009; Okiyoneda et al., 2013). A truncation mutant (F508del/K1218X-CFTR), shown to be misfolded, failed to show correction with $\mathrm{C} 4$, leading the authors of this paper to suggest that NBD2 (only partially removed in the mutant) constituted the $\mathrm{C} 4$ binding site (Okiyoneda et al., 2013). On the other hand, Grove et al. (2009) studied a different NBD2 deletion mutant (E1172XCFTR and F508del/E1172X-CFTR) and found that processing was enhanced with $\mathrm{C} 4$, arguing that NBD2 is not essential for C4 corrector activity. The findings in the current work supported the Grove et al. (2009) study and their interpretation that NBD2 is not critical for C4 activity. Inspection of the recent CFTR protein structural models solved by Liu et al. (2017) and Zhang et al. (2017), suggests that the E1172X-CFTR mutant better recapitulates an NBD2 deletion mutant than K1218X-CFTR (Okiyoneda et al., 2013).

Importantly, we showed that C4 interaction stabilized the MSD2 protein fragment, but not the MSD1 fragment, 

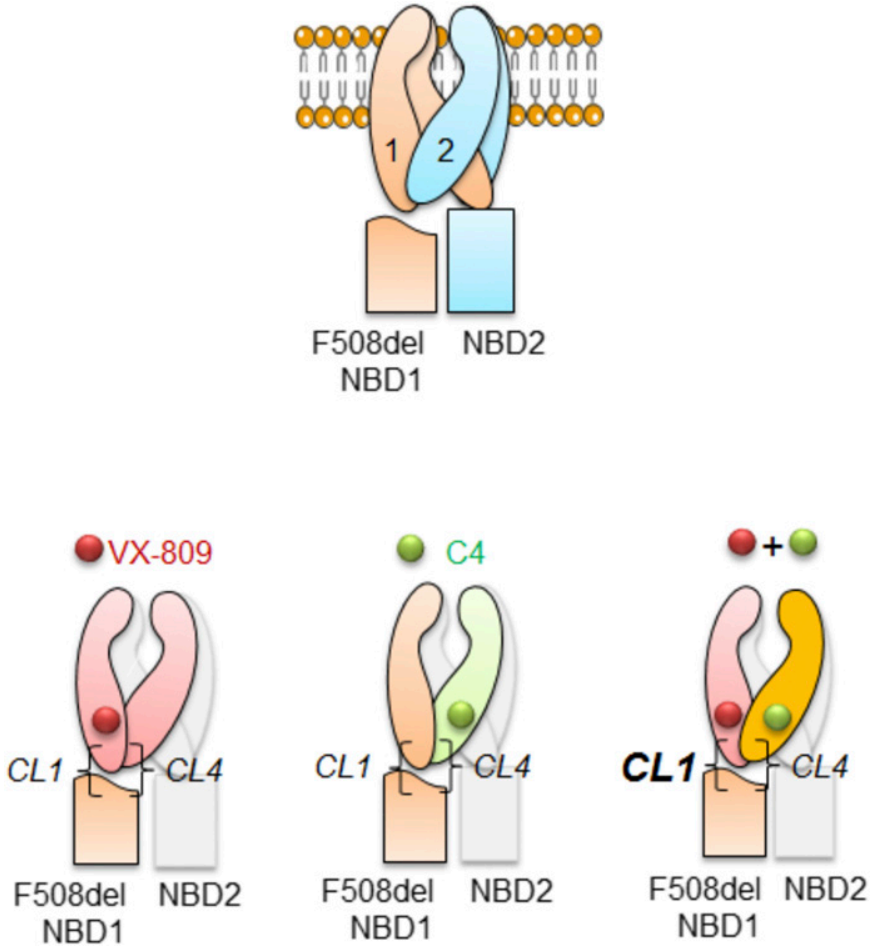

Fig. 5. Graphic hypothesis depicting putative interaction between VX-809 and C4 and key role of ICL1 (CL1) in corrector activity. (Upper panel) The illustration shows the MSD1 and F508del-NBD1 as peach colored and MSD2 and NBD2 as cyan colored. (Lower panel) The illustration on the left-hand side highlights the putative stabilizing effect of VX-809 (red) on MSD1 with its allosteric effect on MSD2. The illustration in the middle shows the putative stabilizing effect of C4 (green) on MSD2. The illustration on the right-hand side shows the putative interaction of VX-809 plus C4 on MSD2 (orange) and the primary role of CL1 on mediating correction by the combination.

suggesting that MSD2 is required for $\mathrm{C} 4$ activity. Altogether, our findings suggest that the two correctors, VX-809 and C4, exert nonidentical but potentially overlapping mechanisms of action.

The current work also highlights the essential role of ICL1 in mediating the corrector activity of VX-809, C4, and the combination of these two modulators. Although both correctors enhanced the interaction between ICL4 and NBD1 in WT-CFTR protein, in the context of F508del-CFTR this conformational change was not sufficient to account for the activity of VX-809 or the corrector combination since the secondary mutation (R1070W) in ICL4 did not abolish their effect in improving processing. However, disruption of ICL1 completely abrogated the effect of correctors individually and in combination. Hence, there is a dominant role of the ICL1: NBD1 interaction in the rescue of F508del-CFTR (Loo and Clarke, 2017). Examination of the recent structures of the fulllength CFTR protein (Liu et al., 2017; Zhang et al., 2017) will enable the generation of new hypotheses regarding the effect of small molecule binding on the ICL1:NBD1 interface.

As previously mentioned, our results suggest distinct binding sites for VX-809 and C4 on the CFTR protein, and support the current hypothesis that the combination of compounds will lead to more effective therapies for subjects bearing the major mutation. Encouraging results are emerging from clinical trials of corrector combinations developed by Vertex Pharmaceuticals (San Diego, CA). One of the compounds is VX-809 (lumacaftor); however, the structure of the second compound is not known. The field is excited to learn in preliminary press releases that the combination is exerting improved clinical responses relative to those observed when a single corrector (i.e., lumacaftor alone) was administered. Soon, we will learn if this clinical promise persists and whether the second corrector resembles the mechanism of action that we have described for $\mathrm{C} 4$.

\section{Acknowledgments}

The authors thank Dr. David Clarke (University of Toronto) for providing the A52-tagged MSD2 construct, mAb A52, as well as the cysteine-less WT-CFTR construct and V510C(NBD1)/A1067C(ICL4)CFTR mutant construct; Bruna Di Benedetto (University of Bari) for technical assistance with chemical crosslinking studies; Dr. Elena Ciani (University of Bari) for helpful discussions and assistance regarding construction of the F508del/R1070W-CFTR mutant; Roberto Gambari (Department Life Sciences and Biotechnology, University of Ferrara, Ferrara, Italy) for providing the small molecule TMA; and Jacqueline McCormack for assistance in the editing of the paper.

\section{Authorship Contributions}

Participated in research design: Laselva, Bear.

Conducted experiments: Laselva.

Performed data analysis: Laselva.

Wrote or contributed to the writing of the manuscript: Laselva, Molinski, Casavola, Bear.

\section{References}

Abbattiscianni AC, Favia M, Mancini MT, Cardone RA, Guerra L, Monterisi S, Castellani S, Laselva O, Di Sole F, Conese M, et al. (2016) Correctors of mutant CFTR enhance subcortical cAMP-PKA signaling through modulating ezrin phosphorylation and cytoskeleton organization. J Cell Sci 129:1128-1140.

Cui L, Aleksandrov L, Chang XB, Hou YX, He L, Hegedus T, Gentzsch M, Aleksandrov A, Balch WE, and Riordan JR (2007) Domain interdependence in the biosynthetic assembly of CFTR. J Mol Biol 365:981-994.

Cutting GR (2015) Cystic fibrosis genetics: from molecular understanding to clinical application. Nat Rev Genet 16:45-56.

D'Antonio C, Molinski S, Ahmadi S, Huan LJ, Wellhauser L, and Bear CE (2013) Conformational defects underlie proteasomal degradation of Dent's diseasecausing mutants of ClC-5. Biochem J 452:391-400.

Du K and Lukacs GL (2009) Cooperative assembly and misfolding of CFTR domains in vivo. Mol Biol Cell 20:1903-1915.

Favia M, Mancini MT, Bezzerri V, Guerra L, Laselva O, Abbattiscianni AC, Debellis L, Reshkin SJ, Gambari R, Cabrini G, et al. (2014) Trimethylangelicin promotes the functional rescue of mutant F508del CFTR protein in cystic fibrosis airway cells. Am J Physiol Lung Cell Mol Physiol 307:L48-L61.

Gadsby DC, Vergani P, and Csanády L (2006) The ABC protein turned chloride channel whose failure causes cystic fibrosis. Nature 440:477-483.

Grove DE, Rosser MF, Ren HY, Naren AP, and Cyr DM (2009) Mechanisms for rescue of correctable folding defects in CFTRAF508. Mol Biol Cell 20:4059-4069.

He L, Kota P, Aleksandrov AA, Cui L, Jensen T, Dokholyan NV, and Riordan JR (2013) Correctors of $\triangle$ F508 CFTR restore global conformational maturation without thermally stabilizing the mutant protein. FASEB $J$ 27:536-545.

Huang P, Gilmore E, Kultgen P, Barnes P, Milgram S, and Stutts MJ (2004) Local regulation of cystic fibrosis transmembrane regulator and epithelial sodium channel in airway epithelium. Proc Am Thorac Soc 1:33-37.

Hudson RP, Dawson JE, Chong PA, Yang Z, Millen L, Thomas PJ, Brouillette CG, and Forman-Kay JD (2017) Direct binding of the corrector VX-809 to human CFTR NBD1: evidence of an allosteric coupling between the binding site and the NBD1: CL4 interface. Mol Pharmacol 92:124-135.

Laselva O, Molinski S, Casavola V, and Bear CE (2016) The investigational cystic fibrosis drug trimethylangelicin directly modulates CFTR by stabilizing the first membrane-spanning domain. Biochem Pharmacol 119:85-92.

Li C and Naren AP (2005) Macromolecular complexes of cystic fibrosis transmembrane conductance regulator and its interacting partners. Pharmacol Ther 108:208-223.

Liu F, Zhang Z, Csanady L, Gadsby DC, and Chen J (2017) Molecular structure of the human CFTR ion channel. Cell 169:85-95.e8.

Loo TW, Bartlett MC, and Clarke DM (2013) Corrector VX-809 stabilizes the first transmembrane domain of CFTR. Biochem Pharmacol 86:612-619.

Loo TW and Clarke DM (2017) Corrector VX-809 promotes interactions between cytoplasmic loop one and the first nucleotide-binding domain of CFTR. Biochem Pharmacol 136:24-31.

Mendoza JL, Schmidt A, Li Q, Nuvaga E, Barrett T, Bridges RJ, Feranchak AP, Brautigam CA, and Thomas PJ (2012) Requirements for efficient correction of $\triangle$ F508 CFTR revealed by analyses of evolved sequences. Cell 148:164-174.

Molinski S, Eckford PD, Pasyk S, Ahmadi S, Chin S, and Bear CE (2012) Functional rescue of F508del-CFTR using small molecule correctors. Front Pharmacol 3:160. 
Molinski SV, Ahmadi S, Hung M, and Bear CE (2015) Facilitating structure-function studies of CFTR modulator sites with efficiencies in mutagenesis and functional screening. J Biomol Screen 20:1204-1217.

Molinski SV, Ahmadi S, Ip W, Ouyang H, Villella A, Miller JP, Lee PS, Kulleperuma K, Du K, Di Paola M, et al. (2017) Orkambi ${ }^{\circledR}$ and amplifier co-therapy improves function from a rare $C F T R$ mutation in gene-edited cells and patient tissue. EMBO Mol Med 9:1224-1243.

Mornon JP, Lehn P, and Callebaut I (2008) Atomic model of human cystic fibrosis transmembrane conductance regulator: membrane-spanning domains and coupling interfaces. Cell Mol Life Sci 65:2594-2612.

Okiyoneda T, Veit G, Dekkers JF, Bagdany M, Soya N, Xu H, Roldan A, Verkman AS, Kurth M, Simon A, et al. (2013) Mechanism-based corrector combination restores $\triangle$ F508-CFTR folding and function. Nat Chem Biol 9:444-454

Rabeh WM, Bossard F, Xu H, Okiyoneda T, Bagdany M, Mulvihill CM, Du K, di Bernardo S, Liu Y, Konermann L, et al. (2012) Correction of both NBD1 energetics and domain interface is required to restore $\Delta$ F508 CFTR folding and function. Cell 148:150-163.

Ren HY, Grove DE, De La Rosa O, Houck SA, Sopha P, Van Goor F, Hoffman BJ, and Cyr DM (2013) VX-809 corrects folding defects in cystic fibrosis transmembrane conductance regulator protein through action on membrane-spanning domain 1. Mol Biol Cell 24:3016-3024.

Riordan JR (2008) CFTR function and prospects for therapy. Annu Rev Biochem 77: $701-726$
Riordan JR, Rommens JM, Kerem B, Alon N, Rozmahel R, Grzelczak Z, Zielenski J, Lok S, Plavsic N, Chou JL, et al. (1989) Identification of the cystic fibrosis gene: cloning and characterization of complementary DNA. Science $\mathbf{2 4 5}$ 1066-1073.

Van Goor F, Hadida S, Grootenhuis PD, Burton B, Cao D, Neuberger T, Turnbull A, Singh A, Joubran J, Hazlewood A, et al. (2009) Rescue of CF airway epithelial cell function in vitro by a CFTR potentiator, VX-770. Proc Natl Acad Sci USA 106. 18825-18830.

Van Goor F, Hadida S, Grootenhuis PD, Burton B, Stack JH, Straley KS, Decker CJ, Miller M, McCartney J, Olson ER, et al. (2011) Correction of the F508del-CFTR protein processing defect in vitro by the investigational drug VX-809. Proc Natl Acad Sci USA 108:18843-18848.

Zhang Z, Liu F, and Chen J (2017) Conformational changes of CFTR upon phosphorylation and ATP binding. Cell 170:483-491.e8.

Address correspondence to: Christine E. Bear, Molecular Medicine, Research Institute, Hospital for Sick Children, Peter Gilgan Centre for Research and Learning, 686 Bay Street, Room 20.9420, Toronto, ON M5G 0A4, Canada. E-mail: bear@sickkids.ca 